\title{
An efficient translation scheme for representing nurse rostering problems as satisfiability problems
}

\author{
Stefaan Haspeslagh ${ }^{1}$, Tommy Messelis ${ }^{1}$, Greet Vanden Berghe ${ }^{2}$ and Patrick De Causmaecker ${ }^{1,2}$ \\ ${ }^{1}$ CODeS (member of ITEC-IBBT-KU Leuven), \\ Department of Computer Science, KU Leuven KULAK, \\ E. Sabbelaan 53, 8500 Kortrijk, Belgium \\ ${ }^{2}$ CODeS, Vakgroep IT, KAHO, Gebr. De Smetstraat 1, 9000 Gent, België \\ \{stefaan.haspeslagh, tommy.messelis,patrick.decausmaecker\}@kuleuven-kulak.be, greet.vandenberghe@kahosl.be
}

\begin{abstract}
Keywords: Nurse Rostering, Satisfiability Problems, Automated Translation, Counting Constraints
Abstract: In this paper we present efficient translation schemes for converting nurse rostering problem instances into satisfiability problems (SAT). We define eight generic constraints types allowing the representation of a large number of nurse rostering constraints commonly found in literature. For each of the generic constraint types, we present efficient translation schemes to SAT. Special attention is paid to the representation of counting constraints. We developed a two way translation scheme for counting constraints using $O($ nlogn $)$ variables and $O\left(n^{2}\right)$ clauses. We translated the instances of the First international nurse rostering competition 2010 to SAT and proved the infeasibility of the instances. The SAT translation was used for a hardness study of nurse rostering problem instances based on SAT features.
\end{abstract}

\section{INTRODUCTION}

We present schemes for automatically translating nurse rostering problem instances into satisfiability problem instances (SAT).

The SAT-translation scheme was originally designed for a hardness study of nurse rostering problem instances (Bilgin et al., 2009). Some algorithms perform well on some specific instances while those same algorithms perform worse on others. (LeytonBrown et al., 2006) present an experimental approach for predicting the running time of algorithms designed to solve the winner determination problem for combinatorial auctions. The previous strategy was applied to the more abstract problem of propositional satisfiability (Nudelman et al., 2004) introducing a set of 91 features for the runtime prediction of several algorithms that prove whether a certain problem instance is satisfiable or not. This work has led to the construction of SATzilla, a portfolio solver for SAT problems (Xu et al., 2008). This portfolio was very successful, winning several tracks of different SAT competitions ${ }^{1}$. The aforementioned SAT-features set is used to predict the runtime of a certain algorithm and the

\footnotetext{
${ }^{1}$ More information about these competitions can be found at http://www. satcompetition.org
}

value of the objective function obtained by the algorithm.

A second potential application of the SAT translation scheme is applying SAT (or MaxSAT) solvers to nurse rostering problem instances. As a first attempt, (Acharyya, 2008) translates a simple nurse rostering problem with a small set of constraints into SAT and applies GSAT to solve it. The approach is limited to small instances as the number of clauses increases rapidly with the size of the instances. It is thus important to develop efficient encodings of the constraints.

Modeling optimisation problems involving constraints on sequences of decision variables to mixed integer programs (and SAT) can be very complex (Côté et al., 2011). Special attention is given to the translation of counting constraints into SAT clauses. We present an efficient translation procedure generating $O\left(n^{2}\right)$ clauses and $O(n \log n)$ variables. (Bailleux and Boufkhad, 2003) developed a translation scheme with similar complexity. (Sinz, 2005) provides a $O(n . k)$ and (Asín et al., 2009) a $O\left(n \log ^{2}(k)\right)$ scheme to translate the constraint $x_{1}+\ldots+x_{n} \leq k$. All contributions however, only provide a one way translation, preserving arc consistency. As for the hardness studies, we do not solely focus on solving nurse rostering problems using SAT, a two way translation was required. 
(Cadoli and Schaerf, 2005) present the automated translation of problem specifications expressed in NPSEC into SAT. NP-SEC in a logic based language capable of expressing all problems belonging to complexity class NP. The authors tested the system on a few classical problems such as graph colouring and job-shop scheduling, NP-complete problems with a rather simple problem definition. The authors highlight the benefits of an automated approach. Although the performance of SAT solvers on the generated instances is often inferior to manual encodings, the authors stem that the system is a valuable tool for developing fast prototypes for new problems, or variations of known ones for which no specific solver is available.

In Section 2, an abstract respresentation of nurse rostering problems using numberings is described. Section 3 elaborates on the formal definition of generic constraints types. In Section 4, each generic constraint type is translated to SAT. Section 5 demonstrates the applicability of the SAT translation schemes for a hardness study of nurse rostering problem instances. Section 6 concludes and gives directions for further research.

\section{Abstract representation of nurse rostering problems using numberings}

The nurse rostering problem under study is the problem presented in the First international nurse rostering competition (Haspeslagh et al., 2012). The authors used numberings, originally designed for the efficient evaluation of constraint violations (Burke et al., 2001) as an abstract representation of constraints. After we elaborate on the definition of numberings, we give an example numbering for some sample constraints. For a full description of the competition's constraint, we refer to the original paper.

\subsection{Numberings}

We start with some elementary notation.

Definition 1. A time unit is an elementary interval of time in which a nurse can be assigned a shift.

In our case, shift types determine those intervals. So, each shift type has a corresponding time unit. In the case of the nurse rostering problems considered within this paper, the number of time units equalled the number of shift types times the number of days in the planning period. Thus, supposing we have a planning horizon of $D$ days and for each day there are
$S h$ shift types, we have a set $T$ of $D * S h$ time units. A solution to a nurse rostering problem is then an assignment of nurses to shifts on specific time units.

Numberings on the time units are defined as follows:

Definition 2. A numbering $N_{i}$ is a mapping of the set of time units onto a set of numbers extended with the symbol $U$ i.e. $\quad N_{i}: T \rightarrow\{-M,-M+$ $1, \ldots, 0,1, \ldots, M-1, M, U\}$ where $i=1, \ldots, I$ and $I$ is the total number of numberings. $M$ is a positive integer and $U$ (undefined) is a symbol introduced to represent the time units that are not mapped onto a number.

The mapping does not need to be into or onto, nor does it need to preserve sequence. An event is a time unit for which a nurse has a shift type assigned. The idea of the evaluation method (Burke et al., 2001) is to go through the set of events for which the time units do not have $U$ (undefined) as value. We call these the 'numbered events'. More formally:

Definition 3. A personal schedule $S_{p}$ for person $p$ is a mapping $S_{p}: T \rightarrow\{$ working, free $\}$.

Definition 4. For a given personal schedule $S_{p}$ an event is a time unit e for which $S_{p}(e)=$ working.

Denote by $T_{S_{p}}$ the set of all time units for which $S_{p}$ maps to working. $T_{S_{p}}$ is thus the set of time units induced by $S_{p}$, or in other words: the set of time units for which nurse $p$ is assigned to work. Denote by $T_{N_{i}}$ the set of time units for which the numbering $N_{i}$ is defined.

Definition 5. The event set $T_{S_{p}, N_{i}}:=T_{S_{p}} \cap T_{N_{i}}$ is the set of time units induced by the schedule $S_{p}$, for which the numbering $N_{i}$ is defined $(\neq U)$.

Definition 6. Two events $e_{j}$ and $e_{j+1}$ are consecutive with respect to numbering $N_{i}$ if $N_{i}\left(e_{j+1}\right)-N_{i}\left(e_{j}\right)=1$

We can now express the following four groups of constraints:

- total number of assignments (total): for a numbering $N_{i}$, this constraint type limits the number of events $e$ for which $N_{i}(e) \neq U$.

- total number of assignments of a certain type (perType): for a numbering $N_{i}$, this constraint type limits the number of events corresponding to a specific number $j\left(N_{i}(e)=j\right)$.

- consecutive assignments (consecutiveness): for a numbering $N_{i}$, this constraint limits the length a a sequence of consecutive events in $T_{S_{p}, N_{i}}$.

- gaps between consecutive sequences of assignments (between): for a numbering $N_{i}$ this constraint type limits the maximum gap (e.g. free time) between two non-consecutive events $e_{j}$ and $e_{j+1}$ (the gap equals $\left.N_{i}\left(e_{j+1}\right)-N_{i}\left(e_{j}\right)\right)$. 


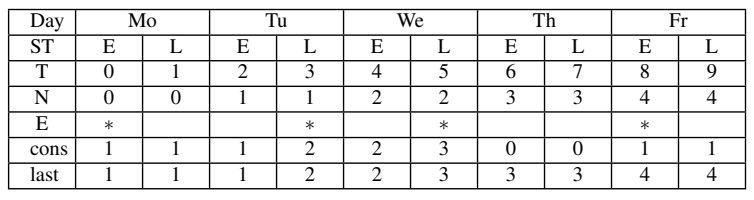

Table 1: Example numbering

For each constraint type, a maximum and minimum bound can be set: max_total and min_total, max_consecutive and min_consecutive, max_between and min_between and max_pert and min_pert.

Four counting variables are introduced. total represents the total number of events for the numbering. consecutive represents the number of consecutive events. If an interruption in a sequence is found, the counter is reset to 0 . pert keeps track of the number of events per value in the numbering and last keeps track of the number of the last evaluated event.

With each numbering $N_{i}$ a set of four of the above counters is associated. During the evaluation, by comparing the counter with one of the abovementioned constraint types, constraint violations are detected.

An example for a simplified nurse rostering problem with two shift types (ST) is given in Table 1 . We want to evaluate two constraints: the employee should work at most two consecutive days (max_consecutive $=2$ ) and should have at least two consecutive days off (min_between $=2$ ). $\quad E$ shows the assignments to one employee. At time unit 5, a first violation is detected as the value of the counter cons exceeds max_consecutive. At time unit 8, a second violation is detected as (current_number-last $>$ min_between).

\section{Formal definitions of generic constraints}

In this study, we only consider monotonically ascending (Definition 7) numberings.

Definition 7. A numbering $N_{i}$ is monotonically ascending if, for every two time units $j_{a}$ and $j_{b}$ for which $N_{i}$ is defined $(\neq U), j_{a}<j_{b} \Rightarrow N_{i}\left(j_{a}\right) \leq N_{i}\left(j_{b}\right)$.

The constraints on the numberings can be expressed using event sets. An important concept in the expression of constraints is the notion of event sequences, in particular sequences that increase at a steady pace.

Definition 8. The event set $E$ of a numbering $N_{i}$ and a personal schedule $S_{p}$ is the set $T_{S_{p}, N_{i}}$.

With every event $e \in E$, a unique number $\left(\in N_{i}\right)$ is associated. We refer to Section 2.1 for detailed information on the definition of $S_{p}$ and $T_{S_{p}, N_{i}}$.
Definition 9. An event sequence $r$ is any sequence of events $e_{j}$ from $E,(j=0 \ldots m)$, conserving the order of the time units corresponding to the events.

Definition 10. A sequence $r$ is contiguously ascending if $\forall j \in\{1, \ldots, m\}: e_{j}-e_{j-1}=1$.

We can now give formal definitions for the constraints in Table 2. Note that the subject of these constraints is depending on the numbering. All constraints of the competition's instances can be described using monotonically ascending numberings and are of one of the presented constraint types.

\begin{tabular}{|c|c|c|}
\hline Constraint & Value & Definition \\
\hline max_consecutive & $\max _{C}$ & $\begin{array}{l}\text { There is no contiguously ascending event se- } \\
\text { quence of length } \max _{C}+1\end{array}$ \\
\hline min_consecutive & $\min _{c}$ & $\begin{array}{l}\text { There is no contiguously ascending event se- } \\
\text { quence of length } l: \min _{c}>l \geq 1 \text { which is not } \\
\text { part of another ascending event sequence of } \\
\text { length at least } \min _{c} \text {. }\end{array}$ \\
\hline max_between & $\max _{b}$ & $\begin{array}{l}\text { For any two consecutive events } e_{j} \text { and } e_{j+1}, \\
N_{i}\left(e_{j+1}\right)-N_{i}\left(e_{j}\right) \leq \max _{b}\end{array}$ \\
\hline min_between & $\min _{b}$ & $\begin{array}{l}\text { For any two consecutive events } e_{j} \text { and } e_{j+1}, \\
N_{i}\left(e_{j+1}\right)-N_{i}\left(e_{j}\right) \leq 1 \text { or } N_{i}\left(e_{j+1}\right)-N_{i}\left(e_{j}\right) \geq \\
\min _{b}\end{array}$ \\
\hline max_total & $\max _{t}$ & The event set $E$ contains at most max $_{t}$ events \\
\hline min_total & $\min _{t}$ & The event set $E$ contains at least $\min _{t}$ events \\
\hline max_perType $(i)$ & $\max _{p t}$ & $\begin{array}{l}\text { The event set } E \text { contains at most } \max _{p t} \text { events } \\
\text { corrensponding to a number } k\end{array}$ \\
\hline min_perType $(i)$ & $\min _{p t}$ & $\begin{array}{l}\text { If the event set } E \text { contains an event correspond- } \\
\text { ing to a number } k \text {, then it contains at least } \min _{p t} \\
\text { events corresponding to } k\end{array}$ \\
\hline
\end{tabular}

Table 2: Formal definition of eight generic constraints

\section{Translation of generic constraints to SAT}

We present a scheme to translate each of the eight generic constraints from Section 3 to Conjunctive Normal Form SAT clauses (Cook, 1971). A preprocessing step is performed before the translation of the constraint types.

\subsection{Preprocessing}

We introduce boolean decision variables $v_{p, j}$ indicating whether nurse $p$ is working time unit $j$. Since the expression of the sequence constraints is basically the same for all employees, we denote $v_{p, j}$ as $v_{j}$, in order to simplify the notation.

For some numberings, consecutive time units are assigned the same number, e.g. the example numbering $N$ in Table 1 does not distinguish between different shifts on the same day. Hence it is natural to introduce a variable $t_{i}$ indicating for each sequence of consecutive time units with equal numbers whether an employee is working on a time unit within that sequence. Generally, for such a sequence of time units starting at $k$ and ending at $l$ : 


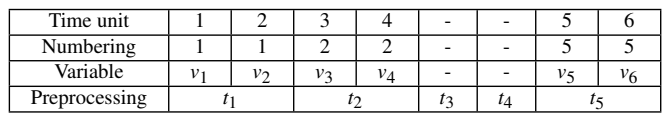

Table 3: Preprocessing for 'between' constraints

$t_{i} \Leftrightarrow \bigvee_{j=k}^{l} v_{j}$ or in cnf: $\neg t_{i} \vee\left(\bigvee_{j=k}^{l} v_{j}\right)$ and $\bigwedge_{j=k}^{l}\left(t_{i} \vee \neg v_{j}\right)$

This results in $2+l-k$ clauses. For $n$ numbers in the numbering we generate at most $\lceil n / 2\rceil$ variables and at most $1+\lceil n / 2\rceil$ clauses. The preprocessing step thus results in $O(n)$ variables and $O(n)$ clauses.

\subsubsection{Preprocessing for between constraints}

Translating 'between' constraints requires a slightly different preprocessing step. Where a numbering is interrupted, one or more implicit variables with value 'False' should be placed. An example is given in Table 3 , both variables $t_{3} \equiv$ False and $t_{4} \equiv$ False. Without the implicit variables, some event sequences would lack. Suppose we want a gap of at most 1 between two consecutive events and an event occurs for $t_{2}\left(v_{3}=\right.$ True or $v_{4}=$ True $)$ and $t_{5}\left(v_{5}=\right.$ True or $v_{6}=$ True). Between the two events, there is a gap of 2. By omitting the implicit variables, the two event sequences detecting the violation would be missing. The preprocessing step results in at most $O(n)$ extra variables and as many extra clauses as variables added.

\subsection{Translation of 'consecutive' and 'between' constraints}

\subsubsection{Maximum number of consecutive events} and free time units between two events.

As stated in Table 2 there should not be a contiguously ascending event sequence of length $\max _{c}+1$. For every contiguously ascending sequence cas ( cas $_{i}$ is the index in the original sequence of the $i^{\text {th }}$ variable within cas) we have the following clauses:

$$
\neg\left(\bigwedge_{j=0}^{\max _{c}} t_{\text {cas }_{i}}\right) \text {, in cnf: } \bigvee_{i=0}^{\max _{c}}\left(\neg t_{\text {cas }_{i}}\right)
$$

Analogously, for a maximum gap between two events, we obtain:

$$
\neg\left(\bigwedge_{j=0}^{\max _{b}} \neg t_{\text {cas }_{i}}\right), \text { in cnf: } \bigvee_{i=0}^{\max _{b}}\left(t_{\text {cas }_{i}}\right)
$$

In general, both constraints for a monotonically ascending numbering with $n$ numbers generates at most $\left(n-\max _{c}\right)$, respectively $\left(n-\max _{b}\right)$ clauses and no extra variables.

\subsubsection{Minimum number of consecutive events and free time units between two events}

Following Table 2, there should not be a contiguously ascending event sequence of length $1\left(\min _{c}>l>1\right)$ which is not part of another contiguously ascending event sequence of length at least $\min _{c}$. This implies that in any consecutive sequence of variables $t_{i}$ of length $1\left(\min _{c}+1 \geq l \geq 3\right)$ the middle variables cannot be true without one of the border variables. For every contiguously ascending sequence $c a s$ of length $l$ $\left(3 \leq l \leq \min _{c}+1\right)$ this results in the following clauses:

$$
\begin{aligned}
& \left.\neg\left(\neg t_{\text {cas }_{0}} \wedge \neg t_{\text {cas }_{l-1}} \wedge\left(\bigwedge_{i=1}^{l-2} t_{\text {cas }_{i}}\right)\right)\right), \\
& \quad \text { in cnf: } t_{\text {cas }_{0}} \vee t_{\text {cas }_{l-1}} \vee\left(\bigvee_{i=1}^{l-2} \neg t_{\text {cas }_{i}}\right)
\end{aligned}
$$

Analogously, for a minimum gap between two events, we obtain:

$$
\begin{gathered}
\left.\neg\left(t_{\text {cas }_{0}} \wedge t_{\text {cas }_{l-1}} \wedge\left(\bigwedge_{i=1}^{l-2} \neg t_{\text {cas }_{i}}\right)\right)\right), \\
\text { in cnf: } \neg t_{\text {cas }_{0}} \vee \neg t_{\text {cas }_{l-1}} \vee\left(\bigvee_{i=1}^{l-2} t_{\text {cas }_{i}}\right)
\end{gathered}
$$

In general, both constraints for a monotonically ascending numbering consisting of $n$ numbers, generate at most $\left(n-\min _{c}\right)$ clauses and no extra variables.

\subsection{Translation of counting constraints}

We developed an efficient general procedure for translating counting constraints into CNF clauses. First we elaborate on the procedure in general. Then we show how to translate total and pert constraint types using this procedure.

\subsubsection{General procedure}

This procedure uses an iterative process of introducing variables and clauses. We start by some elementary definitions.

Definition 11. $V$ is the set of variables $v_{i}$ for which we want to count the number of variables that are assigned true.

Definition 12. $U_{\alpha, \beta}$ is the set of indices (of time units) $k$ between $\alpha$ and $\beta$ for which $v_{k}$ in $V$ is assigned true.

Definition 13. $F_{\alpha, \beta}$ is the set of indices (of time units) $k$ between $\alpha$ and $\beta$ for which $v_{k}$ in $V$ is assigned false. 
We then introduce the variables $u_{i}$, respectively $f_{i}$, denoting whether there are at least $i$ elements in the set $U_{1, n}$, respectively $F_{1, n}$, with $n$ the number of elements in $V$ :

$u_{i} \Leftrightarrow\left|U_{1, n}\right| \geq i, f_{i} \Leftrightarrow\left|F_{1, n}\right| \geq i$ for $i \in\{1, \ldots, n\}$

More generally, we introduce the variables $u_{x, y, z}$ $\left(f_{x, y, z}\right)$ denoting whether there are at least $z$ events in the set $U_{x, y}\left(F_{x, y}\right)$ :

$$
u_{x, y, z} \Leftrightarrow\left|U_{x, y}\right| \geq z \text { and } f_{x, y, z} \Leftrightarrow\left|F_{x, y}\right| \geq z
$$

In the remainder of this section, we only consider the first equivalence in (2). As $u_{i}=u_{1, n, i}$, for representing equivalence (1), we show we only need to translate:

$$
u_{1, n, i} \Rightarrow\left|U_{1, n}\right| \geq i
$$

By contradiction, the other direction of the equivalence is equivalent with

$$
\neg u_{1, n, i} \Rightarrow \neg\left(\left|U_{1, n}\right| \geq i\right)
$$

If no $i$ variables may be assigned true, at least $n-i+1$ variables should be assigned false:

$$
\left(\left|U_{1, n}\right|<i\right) \Leftrightarrow\left(\left|F_{1, n}\right| \geq n-i+1\right) \Leftrightarrow f_{n-i+1}
$$

Using (5), implication (4) becomes:

$$
f_{n-i+1} \Rightarrow\left|F_{1, n}\right| \geq n-i+1
$$

This can be translated in the same way as we translate (3).

\subsubsection{Translation of $u_{1, n, i} \Rightarrow\left|U_{1, n}\right| \geq i$}

First we prove the following equivalence:

$$
u_{1, n, i} \Leftrightarrow\left(u_{1, \frac{n}{2}, k} \vee u_{\frac{n}{2}+1, n, l}\right) \text { for } k+l=i+1 .
$$

Lemma 1. $u_{1, n, i} \Rightarrow\left(u_{1, \frac{n}{2}, k} \vee u_{\frac{n}{2}+1, n, l}\right)$ for $k+l=i+1$

In words, when $U_{1, n}$ contains at least $i$ elements, then $U_{1, \frac{n}{2}}$ contains at least $k$ elements or $U_{\frac{n}{2}+1, n}$ contains at least $l$ elements. We will prove this by contradiction.

Proof. Let $k_{0}$ be the number of elements in $U_{1, \frac{n}{2}}$ and $l_{0}$ the number of elements $U_{\frac{n}{2}+1, n}$, then $i_{0}=k_{0}+l_{0}$ is the number of elements in $U_{1, n}^{2}$.

Suppose that the right part of the implication does not hold, so we have $k_{0}<k$ and $l_{0}<l$

$$
\begin{aligned}
& \left(k_{0}<k\right) \text { and }\left(\quad l_{0}<l\right) \\
& \Leftrightarrow\left(k_{0} \leq k-1\right) \text { and }\left(l_{0} \leq l-1\right) \\
& \Rightarrow\left(k_{0}+l_{0}\right) \leq(k+l-1-1) \\
& \Leftrightarrow \quad i_{0} \quad \leq\left(\begin{array}{c}
i-1 \\
i
\end{array}\right)
\end{aligned}
$$

which is per definition $\neg u_{1, n, i}$ and thus contradicts to the left part of the implication.
Using the above lemma we can split up the event sets and rewrite the definition of $u_{1, n, i}$ as:

$$
\begin{array}{r}
u_{1, n, i} \Rightarrow\left(u_{1, \frac{n}{2}, k} \vee u_{\frac{n}{2}+1, n, l}\right) \quad \text { for all } \quad k \geq 0, l \geq 0 \\
\text { s.t. } \quad k+l=i+1, i \in\{1, \ldots, n\}
\end{array}
$$

This is equivalent to:

$$
\begin{array}{r}
u_{1, n, i} \Rightarrow \bigwedge_{k, l}\left(u_{1, \frac{n}{2}, k} \vee u_{\frac{n}{2}+1, n, l}\right) \quad \text { for all } k \geq 0, l \geq 0 \\
\text { s.t. } \quad k+l=i+1, i \in\{1, \ldots, n\}
\end{array}
$$

This implication is then formulated as a CNF formula: $\bigwedge_{k, l}\left(\neg u_{1, n, i} \vee u_{1, \frac{n}{2}, k} \vee u_{\frac{n}{2}+1, n, l}\right) \quad$ for all $\quad k \geq 0, l \geq 0$

$$
\text { s.t. } \quad k+l=i+1
$$

Given the limitations we can find $(i+2)$ pairs of values for $\mathrm{k}$ and 1 . Hence we need a total of $(i+2)$ clauses to represent this implication as a cnf formula. This procedure essentially expresses the variable $u_{1, n, i}$ in terms of lower level variables $u_{1, \frac{n}{2}, k}$ and $u_{\frac{n}{2}+1, n, l}$. We need $2(i+2)$ lower level variables to represent the clauses. We must note here that we do not always need all of these lower level variables, indeed the variables $u_{1, \frac{n}{2}, z}$ and $u_{\frac{n}{2}+1, n, z}$ with $z>\left(\frac{n}{2}+1\right)$ will trivially be false, since there can not be more than $\left(\frac{n}{2}+1\right)$ indices in the sets $U_{1, \frac{n}{2}}$ or $U_{\frac{n}{2}+1, n}$. In general, a variable $u_{x, y, z}$ with $z>(y-x+1)$ will always be trivially false. This brings the actual number of lower level to at most $2\left(\frac{n}{2}+1\right)$.

Generally, we need $\min ((n+2), 2(i+2))$ lower level variables. In the worst case, when $i$ equals $n$, this is $(n+2)$.

Whereas the original definition of $u_{i}$ used sets of size $n$, we are now left with sets of size $\frac{n}{2}$. We repeat this recursively until the sets are of size 1 . The variables $u_{x, x, 0}$ and $u_{x, x, 1}$ then correspond to $\neg v_{x}$ and $v_{x}$ respectively.

The 'left' variables $u_{1, \frac{n}{2}, k}$ with different $k$ will in their turn all use the same lower level variables $u_{1, \frac{n}{4}, k^{\prime}}$

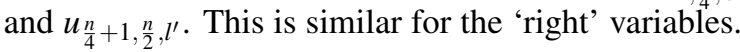
The second iteration will thus introduce $2 .(n / 2+2)$ lower level variables. The total process will ultimately introduce the following number of lower level variables, for $2^{k}=n$ :

$$
\begin{aligned}
(n+2)+2(n / 2+2)+\ldots+2^{k-1}\left(n / 2^{k-1}+2\right) \\
=(n+2)+n+4+\ldots+\quad n+2 n
\end{aligned}
$$

which equals

$$
\begin{aligned}
& (n+n+\cdots+n)+2\left(2^{0}+2^{1}+2^{2}+\cdots+2^{k-1}\right) \\
& \text { with twice } k=\log _{2} n \text { terms } \\
& =n \log _{2} n+\sum_{i=1}^{k}\left(2^{i}\right) \\
& =n \log _{2} n+2 n-2 \\
& =O(n \log n+n) \\
& =O(n \log n)
\end{aligned}
$$


The first iteration introduces $(i+2)$ lower level clauses. In the worst case $i$ equals $n$, thus $(n+2)$ clauses are introduced. In a formula, the set of $k$ and $l$ variables will produce the following number of clauses

$$
\sum_{i=0}^{\lfloor n / 2\rfloor}(i+1)+\sum_{i=0}^{\lceil n / 2\rceil}(i+1)
$$

which is $O\left(n^{2}\right)$.

\subsubsection{Translation of total and perType constraints}

For an employee $\mathrm{p}$ and numbering $N_{x}$, the total constraints impose restrictions on the minimum and maximum number of decision variables $v_{p, j}$ (for which $N_{x}$ is defined) that can be assigned true in a given personal schedule $S_{p}$. Definition 11 to 13 can (assuming $V$ containts $n$ elements) be rewritten:

$$
\begin{aligned}
V & =\left\{v_{p, i} \mid i \in T_{N_{x}}\right\} \\
U_{\alpha, \beta} & =\left\{k \mid v_{p, k} \wedge k \in T_{N_{x}} \wedge(\alpha \leq k \leq \beta)\right\} \\
F_{\alpha, \beta} & =\left\{k \mid \neg v_{p, k} \wedge k \in T_{N_{x}} \wedge(\alpha \leq k \leq \beta)\right\}
\end{aligned}
$$

A min_total $=\min _{t}$ constraint can be translated to SAT by using the general procedure from the previous section to express:

$$
u_{\min _{t}} \Leftrightarrow\left|U_{1, n}\right| \geq \min _{t}
$$

A max_total $=$ max $_{t}$ constraint means at least $n-$ $\max _{t}$ variables should be assigned false. Analogously, this can be expressed by translating

$$
f_{n-\max _{t}} \Leftrightarrow\left|F_{1, n}\right| \geq n-\max _{t}
$$

The translation of pertType constraints is similar. Only difference is that the set of variables $V$ is limited to those corresponding to a specific value $j$.

$$
V=\left\{v_{p, i} \mid i \in T_{N_{x}} \wedge N_{x}(i)=j\right\}
$$

\section{Application: hardness study for nurse rostering problem instances}

Empirical hardness can be understood as the complexity of a problem instance, when solved with a particular solution method, measured by some performance criteria. This hardness is a combination of the intrinsic hardness of the instance and the quality of the procedure on this specific instance. This implies that empirical hardness is inherently linked to the algorithm that is used. Empirical hardness models typically map a set of instance features onto a performance criterion such as the running time of an algorithm.
Instance features are often derived from expert knowledge. However, experts may be biased and good experts in the problem domain may not always be available. Therefore, by translating the problem instances under study to SAT, we examine the feasibility of a more general approach based on well known SAT-features (Nudelman et al., 2004).

For the hardness studies presented in this paper, we follow the methodology presented by (LeytonBrown et al., 2006). Six steps are to be followed:

1. Identification of the problem instance distribution.

2. Selection of one or more algorithms and performance criteria.

3. Selection of a set of inexpensive, distribution independent features.

4. Sampling the instance distribution for generating a training set.

5. Elimination of redundant and uninformative features.

6. Learning models that map the feature space onto the performance criteria.

As hardness studies are not the scope of this paper, we refer to the aforementioned paper for more details on the method used and the technical report (Messelis et al., 2012) for specific and detailed information on the above steps in the light of the nurse rostering problem. We present the results of two performed experiments to demonstrate the applicability of the SAT translation schemes presented in this paper.

The first experiment focusses on the applicability of the SAT features proposed in (Nudelman et al., 2004). We designed a problem instance distribution consisting of "smaller" problem instances" 2 .

The second experiment demonstrates the applicability of hardness models to real world like optimisation problems. Testbed were randomisations of the instances found in the First international nurse rostering competition (Haspeslagh et al., 2012).

\subsection{Applicability of hardness studies using SAT features for NRP}

As stated before, a problem distribution of "smaller" instances is generated. This allows the use of IBM CPLEX to solve the instances. Besides an optimal solver, a variable neighbourhood metaheuristic algorithm (Burke et al., 2008) is applied.

\footnotetext{
${ }^{2}$ Smaller denotes a small planning horizon, a limited number of employees available and a limited number of shift to be covered.
} 


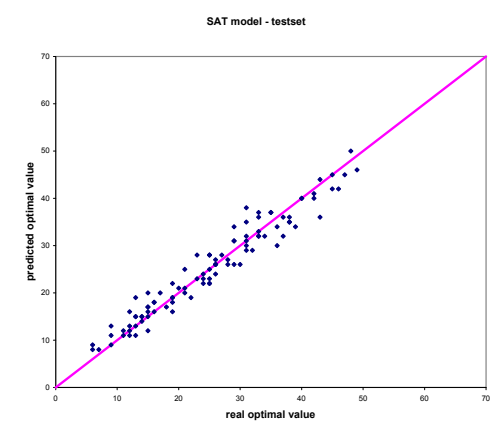

Figure 1: Prediction of the optimal solution quality

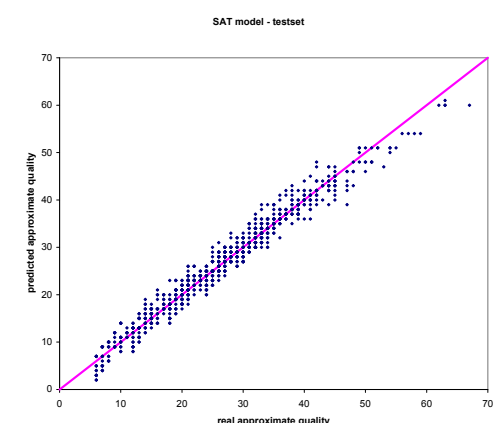

Figure 2: Prediction of the solution quality obtained by the metaheuristic

Using a selected set of features ${ }^{3}$, we were able to predict the quality of the optimal solution and the quality of the approximate solution. Figure 1 shows a plot of the real optimal objective function values versus the predicted values on the test instances. We found as correlation coefficient: $R^{2}=0.99$. Figure 2 shows a plot of the actual metaheuristic objective function values versus the predicted values. A correlation coefficient $R^{2}=0.96$ is obtained.

\subsection{SAT-based hardness analysis for "real world" NRP}

The initial experiment served as a quick proof-ofconcept study to check the applicability of the approach to a small optimisation problem. The same ideas were applied in the context of larger, real world like optimisation problem instances as found in the nurse rostering competition (Haspeslagh et al., 2012). The feature set and the used algorithm are the same as in the previous experiment, the performance criterion under study is the quality of the approximate solution obtained by the metaheuristic. Because of the larger sizes of the instances, compared to the ones of the previous experiment, the use IBM CPLEX is

\footnotetext{
${ }^{3}$ Details on the features can be found in the technical report (Messelis et al., 2012)
}

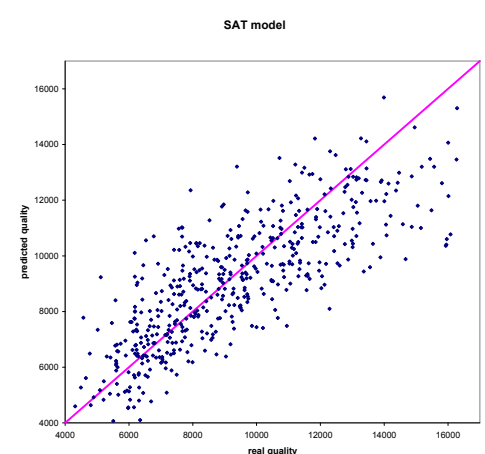

Figure 3: Prediction of the solution quality obtained by the metaheuristic

no longer feasible. Figure 3 shows a plot of the objective function values obtained by the metaheuristic versus the predicted values. A correlation coefficient of $R^{2}=0.985$ is obtained. This coefficient indicates the predictive power of the model is high.

\section{Conclusion \& Further research}

The main contribution of this paper is the automated translation of nurse rostering problem instances into SAT problems instances. To support automated translation, a formal description of nurse rostering problem instances is required. We used numberings (Burke et al., 2001) for clearly and unambiguously describing instances (Haspeslagh et al., 2012). We defined eight generic constraint types allowing the representation of a large number of real world constraints found in literature. For each constraint type, we presented efficient translation schemes to SAT. The preprocessing step for both the SAT translation introduces $O(n)$ variables and $O(n)$ clauses. The SAT translation of the consecutiveness and between constraints generates $O(n)$ clauses. The SAT translation of counting constraints generates $O\left(n^{2}\right)$ clauses and $O(n \log n)$ variables. Summarized, all constraints that can be expressed as monotonic ascending numberings can be translated to SAT. The automated translation allows for an error safe representation of nurse rostering problem instances as instances of other problem domains. A manual translation can be very time consuming and complete equivalence between the original and the translated model may be hard to attain.

This approach allows studying problems from an other point of view. As shown in Section 5, the SAT translation scheme was successfully used to study the hardness of nurse rostering problem instances.Although the aims of the algorithms for which the SAT feature set (Nudelman et al., 2004) was de- 
veloped is very different from the aims of algorithms within the field of nurse rostering research (satisfiability versus optimality), the relevance of SAT features for the hardness analysis of nurse rostering problem instances was demonstrated. As we translate to SAT, information on the objective function of the original problem is lost. As a natural extension, the SAT translation scheme can be adapted to produce MAX-SAT instances thereby 'incorporating information on the objective function'. In a first effort, MAXSAT solvers (Argelich and Manyà, 2006) can be applied to study the solution quality obtained by those solvers. Another research direction is to design hybrid solvers. Current efforts first try to solve a partial problem with an exact solver (Burke and Curtois, 2011; Valouxis et al., 2012). The obtained solution is then optimised using for example a metaheuristic. One interesting research challenge is to study the opposite. SAT solvers are able to search the entire solution space. A metaheuristic search method only explores the solution space partially. In a sense, metaheuristics are designed for trying to escape local optima in the solution space, e.g. a metaheuristic is used to incorporate diversification in the search process. By adding extra constraints, based on the solutions obtained by a metaheuristic search method, we can force the (MAX-)SAT solvers not to explore those parts of the solution space covered by the metaheuristic search and therefore try to intensify diversification.

\section{REFERENCES}

Acharyya, S. (2008). A SAT Approach for Solving The Nurse Scheduling Problem. In IEEE Region 10 Conference.

Argelich, J. and Manyà, F. (2006). Exact max-sat solvers for over-constrained problems. Journal of Heuristics, 12:375-392.

Asín, R., Nieuwenhuis, R., Oliveras, A., and RodríguezCarbonell, E. (2009). Cardinality networks and their applications. In Proceedings of the 12th International Conference on Theory and Applications of Satisfiability Testing, SAT '09, pages 167-180.

Bailleux, O. and Boufkhad, Y. (2003). Efficient CNF Encoding of Boolean Cardinality Constraints. In Rossi, F., editor, Principles and Practice of Constraint Programming - CP 2003, volume 2833 of Lecture Notes in Computer Science, pages 108-122. Springer Berlin / Heidelberg.

Bilgin, B., De Causmaecker, P., Haspeslagh, S., Messelis, T., and Vanden Berghe, G. (2009). Hardness studies for nurse rostering problems. In LION, Trento, Italy, 14-18 January 2009.

Burke, E. and Curtois, T. (2011). New computational results for nurse rostering benchmark instances. technical re- port, 2011. Technical report, School of Computer Science, University of Nottingham.

Burke, E. K., Curtois, T., Post, G., Qu, R., and Veltman, B. (2008). A hybrid heuristic ordering and variable neighbourhood search for the nurse rostering problem. European Journal of Operational Research, 188(2):330 - 341 .

Burke, E. K., De Causmaecker, P., Petrovic, S., and Vanden Berghe, G. (2001). Fitness Evaluation for Nurse Scheduling Problems. In Proceedings of the Congress on Evolutionary Computation (CEC2001), pages 1139-1146.

Cadoli, M. and Schaerf, a. (2005). Compiling problem specifications into SAT. Artificial Intelligence, 162(12):89-120.

Cook, S. A. (1971). The complexity of theorem-proving procedures. In Proceedings of the third annual ACM symposium on Theory of computing, STOC '71, pages 151-158.

Côté, M.-C., Gendron, B., Quimper, C.-G., and Rousseau, L.-M. (2011). Formal languages for integer programming modeling of shift scheduling problems. Constraints, 16(1):54-76.

Haspeslagh, S., DeCausmaecker, P., Schaerf, A., and Stlevik, M. (2012). The first international nurse rostering competition 2010. Annals of Operations Research, pages 1-16. 10.1007/s10479-012-1062-0.

Leyton-Brown, K., Nudelman, E., and Shoham, Y. (2006). Learning the empirical hardness of optimization problems: The case of combinatorial auctions. In Van Hentenryck, P., editor, Principles and Practice of Constraint Programming - CP 2002, volume 2470 of Lecture Notes in Computer Science, pages 91-100. Springer Berlin / Heidelberg.

Messelis, T., Haspeslagh, S., Vanden Berghe, G., and De Causmaecker, P. (2012). Hardness studies for nurse rostering problems using sat features. Technical report, CODeS, Department of Computer Science, KU Leuven KULAK.

Nudelman, E., Leyton-Brown, K., Hoos, H., Devkar, A., and Shoham, Y. (2004). Understanding random sat: Beyond the clauses-to-variables ratio. In Wallace, M., editor, Principles and Practice of Constraint Programming - CP 2004, volume 3258 of Lecture Notes in Computer Science, pages 438-452. Springer Berlin / Heidelberg.

Sinz, C. (2005). Towards an optimal cnf encoding of boolean cardinality constraints. In Proceedings of the 11th International Conference on Principles and Practice of Constraint Programming (CP 2005), pages 827-831.

Valouxis, C., Gogos, C., Goulas, G., Alefragis, P., and Housos, E. (2012). A systematic two phase approach for the nurse rostering problem. European Journal of Operational Research, 219(2):425 - 433.

Xu, L., Hutter, F., Hoos, H. H., and Leyton-Brown, K. (2008). Satzilla: portfolio-based algorithm selection for sat. J. Artif. Int. Res., 32(1):565-606. 